\title{
Recombinant prion protein induces a new transmissible prion disease in wild-type animals
}

\author{
Natallia Makarava $\cdot$ Gabor G. Kovacs $\cdot$ Olga Bocharova • \\ Regina Savtchenko $\cdot$ Irina Alexeeva $\cdot$ Herbert Budka $\cdot$ \\ Robert G. Rohwer $\cdot$ Ilia V. Baskakov
}

Received: 17 December 2009/Revised: 22 December 2009/ Accepted: 22 December 2009/Published online: 6 January 2010

(C) The Author(s) 2010. This article is published with open access at Springerlink.com

\begin{abstract}
Prion disease is a neurodegenerative malady, which is believed to be transmitted via a prion protein in its abnormal conformation $\left(\mathrm{PrP}^{\mathrm{Sc}}\right)$. Previous studies have failed to demonstrate that prion disease could be induced in wild-type animals using recombinant prion protein $(\mathrm{rPrP})$ produced in Escherichia coli. Here, we report that prion infectivity was generated in Syrian hamsters after inoculating full-length $\mathrm{rPrP}$ that had been converted into the cross- $\beta$-sheet amyloid form and subjected to annealing. Serial transmission gave rise to a disease phenotype with highly unique clinical and neuropathological features. Among them were the deposition of large $\mathrm{PrP}^{\mathrm{Sc}}$ plaques in
\end{abstract}

Electronic supplementary material The online version of this article (doi:10.1007/s00401-009-0633-x) contains supplementary material, which is available to authorized users.

N. Makarava $\cdot$ O. Bocharova $\cdot$ R. Savtchenko $\cdot$

I. V. Baskakov ( $\square)$

Medical Biotechnology Center,

University of Maryland Biotechnology Institute,

725 W. Lombard St., Baltimore, MD 21201, USA

e-mail: Baskakov@umbi.umd.edu

G. G. Kovacs - H. Budka

Institute of Neurology, Medical University of Vienna,

AKH 4J, 1097 Vienna, Austria

I. Alexeeva · R. G. Rohwer

Medical Research Service, Veterans Affairs Maryland Health

Care System, 10 North Greene Street,

Baltimore, MD 21201, USA

R. G. Rohwer

Department of Neurology, University of Maryland,

Baltimore, MD 21201, USA

I. V. Baskakov

Department of Biochemistry and Molecular Biology,

University of Maryland, Baltimore, MD 21201, USA subpial and subependymal areas in brain and spinal cord, very minor lesioning of the hippocampus and cerebellum, and a very slow progression of disease after onset of clinical signs despite the accumulation of large amounts of $\mathrm{PrP}^{\mathrm{Sc}}$ in the brain. The length of the clinical duration is more typical of human and large animal prion diseases, than those of rodents. Our studies establish that transmissible prion disease can be induced in wild-type animals by inoculation of $\mathrm{rPrP}$ and introduce a valuable new model of prion diseases.

Keywords Prion disease - Generating prion infectivity · Prion strains · Prion neuropathology ·

Recombinant prion protein · Amyloid fibrils $\cdot$ Prion plaques

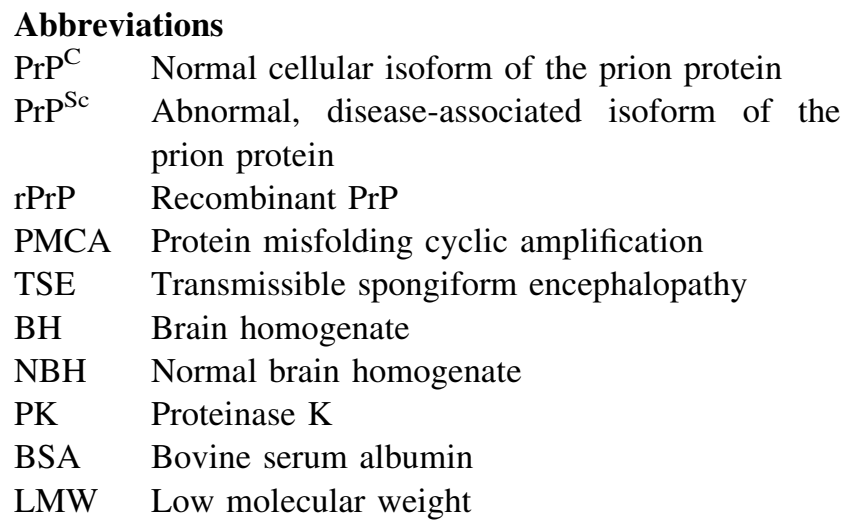

\section{Introduction}

Recent years have witnessed significant progress in providing strong support to the protein-only hypothesis that postulates that the prion diseases are transmitted via a prion protein that acquires an infectious self-replicating 
conformation $\left(\mathrm{PrP}^{\mathrm{Sc}}\right)$ [26]. Previously, amyloid fibrils prepared in vitro from truncated recombinant $\operatorname{PrP}(\mathrm{rPrP})$ [4] were shown to induce transmissible disease in transgenic mice that express the same truncated cellular isoform of the prion protein $\left(\operatorname{PrP}^{C}\right)$ at high levels [20]. However, the origin of transmissible prions in transgenic lines has been disputed due to concerns that the mice expressing $\operatorname{PrP}^{\mathrm{C}}$ at high levels are prone to neurological disorders with undetectably low levels of infectivity. While these concerns have been addressed [9], the objective of inducing prion disease in wild-type animals with $\mathrm{rPrP}$ still represents a great challenge.

Using an alternative approach that involves protein misfolding cyclic amplification (PMCA) conducted in crude brain homogenates, amplification of $\operatorname{PrP}^{\mathrm{Sc}}$ was shown to be accompanied by an amplification of infectivity in vitro [8]. PMCA was also used to successfully generate prion infectivity in vitro de novo from minimal components including $\operatorname{PrP}^{\mathrm{C}}$ and polyanion molecules [11]. Attempts to replace $\operatorname{PrP}^{\mathrm{C}}$ with $\mathrm{rPrP}$ in $\mathrm{PMCA}$, however, have failed, as rPrP was shown to inhibit PMCA reactions [24]. To date, attempts to generate transmissible prion disease in wildtype animals using rPrPs have not been successful despite numerous attempts in which $\mathrm{rPrP}$ was converted into a broad array of abnormal $\beta$-sheet rich conformations.

Here, we report that prion infectivity can be produced in Syrian hamsters by inoculating full-length $\mathrm{rPrP}$ converted into a cross- $\beta$-sheet amyloid conformation and subjected to an annealing procedure [6]. The clinical disease, while clearly recognizable as a transmissible spongiform encephalopathy (TSE) infection, nevertheless exhibited a highly unique clinical course and neuropathological features including large subependymal plaques, minor involvement of cerebellum and hippocampus, but consistent lesioning in thalamus and hypothalamus. Despite large amounts of $\mathrm{PrP}^{\mathrm{Sc}}$ found in the brains, the new disease is characterized by a long clinical duration and very slow progression after the first clinical signs are observed, a feature that is more typical of human and large animal TSEs, than those of rodents.

\section{Materials and methods}

\section{Expression and purification of $\mathrm{PrP}$}

Golden Syrian hamster full-length recombinant PrP encompassing residues 23-231 (rPrP) was expressed and purified according to the previously described procedure [5] with minor modifications. To eliminate adduct formation and increase protein yield, $5 \mathrm{mM}$ DTT instead of $10 \mathrm{mM} \beta$-mercaptoethanol was used to solubilize inclusion bodies. Elution of PrP from the Ni-charged Sepharose resin was performed with $200 \mathrm{mM}$ Imidasole. Oxidative refolding prior to HPLC was achieved by overnight dialysis of IMAC-purified PrP into $8 \mathrm{M}$ Urea, $100 \mathrm{mM}$ Tris, $\mathrm{pH}$ 7.5. During the last $3 \mathrm{~h}$ of dialysis, $10 \mathrm{mM}$ EDTA was added to the dialysis buffer.

\section{Formation of rPrP fibrils}

Lyophilized $\mathrm{rPrP}$ was dissolved in $6 \mathrm{M} \mathrm{GdnHCl}$ to prepare a $3 \mathrm{mg} / \mathrm{ml}$ stock solution of $\mathrm{rPrP}$. To form fibrils, the $\mathrm{rPrP}$ stock solution was diluted to a final protein concentration of $0.5 \mathrm{mg} / \mathrm{ml}$ and incubated at $37^{\circ} \mathrm{C}$ in $20 \mathrm{mM}$ sodium acetate (pH 5.0), $1 \mathrm{M} \mathrm{GdnHCl,} 3 \mathrm{M}$ urea, $150 \mathrm{mM} \mathrm{NaCl}$, $10 \mathrm{mM}$ EDTA for 4 days with continuous horizontal shaking at $600 \mathrm{rpm}$. Amyloid formation was confirmed by increased Thioflavin $\mathrm{T}$ fluorescence and electron microscopy [5]. Fibrils were dialyzed into $10 \mathrm{mM}$ sodium acetate, pH 5.0.

Annealing of fibrils and inoculum preparation

The annealing was performed in two different ways: either in the presence of normal brain homogenate $(\mathrm{NBH})$ or bovine serum albumin (BSA). The NBH used for annealing of rPrP fibrils was prepared from a 25-day-old Syrian hamster, in a facility and with equipment that has never been exposed to TSE agents. The brain was weighed, mixed with nine parts of ice-cold PBS, pH 7.2, and homogenized with a Branson Sonifier-450 tip sonicator until a homogeneous solution was formed (4 pulses of $15 \mathrm{~s}$ sonication with $15 \mathrm{~s}$ cooling on ice between the pulses). After incubation on ice for $30 \mathrm{~min}$, the homogenate was transferred to a new tube leaving behind any crude material that had settled.

rPrP fibrils at $0.1 \mathrm{mg} / \mathrm{ml}$ were heat-treated in PBS, $\mathrm{pH}$ 7.4, in the presence of $5 \% \mathrm{NBH}$ or $5 \mathrm{mg} / \mathrm{ml}$ BSA (Sigma, \#A3294) and $0.1 \%$ Triton X-100 using the following procedure: $50 \mu \mathrm{l}$ aliquots in thin-wall PCR tubes with domed caps were placed into the PCR machine and subjected to 5 cycles of $1 \mathrm{~min}$ incubation at $80^{\circ} \mathrm{C}$ followed by $1 \mathrm{~min}$ incubation at $37^{\circ} \mathrm{C}$. The combined volume was immediately transferred to the animal facility for inoculation. To verify the result of annealing, an aliquot of the inoculum


jected to digestion with $50 \mu \mathrm{g} / \mathrm{ml} \mathrm{PK}$ for $1 \mathrm{~h}$ at $37^{\circ} \mathrm{C}$. PKresistant material was detected by Western immunoblotting with 3F4 antibody.

\section{Bioassay}

For the first passage, weanling golden Syrian hamsters were inoculated intracerebrally with the preparation of $r P r P$ fibrils described above. Each animal was anesthetized with interperitoneal pentobarbital before receiving $50 \mu \mathrm{l}$ of inoculum. Hamsters were observed daily for disease 
starting from the third month postinoculation. Two out of eight inoculated hamsters died intercurrently at 409 and 652 days postinoculation without signs of any disease. The six remaining hamsters were still healthy at 661 days postinoculation when they were euthanized and their brains removed aseptically and saved for subsequent passage and analysis.

For the second passage, $10 \%$ BHs prepared by sonication in PBS, $\mathrm{pH} 7.4$, were dispersed by an additional $30 \mathrm{~s}$ of sonication immediately before inoculation. Each hamster received $50 \mu \mathrm{l}$ of inoculum intracerebrally under general anesthesia ( $2 \% \mathrm{O}_{2} / 4$ MAC isoflurane). After inoculation, hamsters were observed daily for disease.

\section{Proteinase $\mathrm{K}$ resistance assay}

Brains were collected aseptically and cut in half with disposable scalpels. One half was used to prepare $10 \%$ BHs, while the second half was stored at $-80^{\circ} \mathrm{C}$ for future analysis or fixed in formalin for histopathology. Homogenization was performed on ice, in PBS, $\mathrm{pH}$ 7.4, with Sonics vibra cell VCX750 (Newtown, CT) tip sonicator fitted with a stepped microtip by 2 pulses of $30 \mathrm{~s}$ sonication with $30 \mathrm{~s}$ cooling on ice between the pulses. An aliquot of $10 \%$ brain homogenate was mixed with an equal volume of $4 \%$ sarcosyl in PBS, supplemented with $50 \mathrm{mM}$ Tris, $\mathrm{pH} 7.5$, and digested with $20 \mu \mathrm{g} / \mathrm{ml}$ PK for $30 \mathrm{~min}$ at $37^{\circ} \mathrm{C}$ with $1,000 \mathrm{rpm}$ shaking (Eppendorf thermomixer). The reaction was stopped by adding $2 \mathrm{mM}$ PMSF and SDS sample buffer. Samples were boiled for $10 \mathrm{~min}$ and loaded onto NuPAGE 12\% BisTris gels. After transfer to PVDF membrane, PrP was detected with R1/R2 or 3F4 antibody, as indicated. To achieve a comparable intensity of bands, samples without PK were usually loaded at a 1:4 to 1:20 dilution as indicated in the figure legends.

To analyze PK resistance as a function of PK concentration, 10\% BHs prepared by sonication in PBS (see above) were diluted to $1 \%$ using $1 \% \mathrm{BH}$ from healthy hamsters in PBS, pH 7.5, $0.15 \mathrm{M} \mathrm{NaCl}, 1 \%$ Triton, and $0.25 \%$ SDS. Lyophilized Proteinase K (Sigma P6556) was dissolved in the PK buffer containing $20 \mathrm{mM}$ Tris, $\mathrm{pH} 7.5$, and $1 \mathrm{mM} \mathrm{CaCl}_{2}$, and the same buffer was used to prepare serial dilutions of PK. Each aliquot of brain homogenate was supplemented with the same volume, but increasing concentration of $\mathrm{PK}$, with the final concentration ranging from 0 to $2.5 \mathrm{mg} / \mathrm{ml}$. After incubation at $37^{\circ} \mathrm{C}$ for $1 \mathrm{~h}$, the reaction was stopped by addition of SDS-sample buffer and boiling the samples for $10 \mathrm{~min}$. Samples were loaded onto NuPAGE $12 \%$ BisTris gels, transferred to PVDF membrane, and detected with 3F4 antibody. To generate average curves and standard deviation errors, all samples were assayed at least twice.
Deglycosylation of $\mathrm{PrP}^{\mathrm{Sc}}$

Removal of N-linked glycans was performed as previously described [10], using glycerol-free PNGase F and supplied buffers (New England BioLabs). After termination of PK digestion with $2 \mathrm{mM}$ PMSF, the resulting $36 \mu$ of digest was supplemented with $4 \mu \mathrm{l}$ of $10 \times$ glycoprotein buffer and heated at $95^{\circ} \mathrm{C}$ for $10 \mathrm{~min}$. After heat-treatment, $5 \mu \mathrm{l}$ of $10 \times$ G7 reaction buffer, $5 \mu \mathrm{l}$ of $10 \%$ NP-40 and $3 \mu \mathrm{l}$ of PNGase F enzyme were added, and the samples were incubated overnight at $37^{\circ} \mathrm{C}$. The next day, the samples were boiled for $10 \mathrm{~min}$ in SDS sample buffer (Invitrogen) and loaded onto NuPAGE 12\% BisTris gels. After transfer to PVDF, PrP was detected with 3F4 antibody.

\section{Conformational stability assay}

$10 \%$ brain homogenates were incubated with various concentrations of $\mathrm{GdnHCl}$ for $2 \mathrm{~h}$ at room temperature. After incubation, all samples were diluted with $2 \%$ Sarcosyl and PBS to the final concentration of $\mathrm{GdnHCl}$ of $0.4 \mathrm{M}$, digested with $20 \mu \mathrm{g} / \mathrm{ml} \mathrm{PK}$ for $1 \mathrm{~h}$ at $37^{\circ} \mathrm{C}$ with shaking at $1,000 \mathrm{rpm}$ (Eppendorf thermomixer). The reaction was stopped by $2 \mathrm{mM}$ PMSF. After addition of a proteinase inhibitors cocktail (Complete, EDTA-free, Roche), samples were precipitated by $0.3 \%$ sodium phosphotungstate, $\mathrm{pH} 7.4$ as previously described [27]. After overnight incubation at $37^{\circ} \mathrm{C}$ with shaking $1,000 \mathrm{rpm}$ (Eppendorf thermomixer), samples were centrifuged for $30 \mathrm{~min}$ at 13,000 rpm (accuSpin microR centrifuge, Fisher Scientific). The pellet was resuspended in $0.2 \%$ Sarcosyl/ PBS, followed by addition of SDS sample buffer and shaking for $15 \mathrm{~min}$ at $99^{\circ} \mathrm{C}, 1,000 \mathrm{rpm}$. Samples were loaded into NuPAGE $12 \%$ BisTris gels, transferred to PVDF membrane, and detected with 3F4 antibody. Signal intensities were calculated using WCIF ImageJ. The experimental data were fitted using a two-state model and $\mathrm{C}_{50}$ values calculated from fitting parameters. To generate average values and standard deviation errors, all samples were assayed twice.

\section{Protein misfolding cyclic amplification}

Substrate was prepared following the protocol kindly provided by J. Castilla. Healthy hamsters were euthanized and immediately perfused with PBS, pH 7.4, supplemented with $5 \mathrm{mM}$ EDTA. Brains were dissected, $10 \%$ brain homogenate $(\mathrm{w} / \mathrm{v})$ was prepared using ice-cold conversion buffer and glass/Teflon tissue grinders cooled on ice and attached to a constant torque homogenizer (Heidolph RZR2020). The brains were ground at low speed until homogeneous, then 5 additional strokes completed the homogenization. The composition of conversion buffer 
was as previously described [8]: $\mathrm{Ca}^{2+}$ - and $\mathrm{Mg}^{2+}$-free PBS, pH 7.5, supplemented with $0.15 \mathrm{M} \mathrm{NaCl}, 1.0 \%$ Triton, and 1 tablet of complete protease inhibitors cocktail (Roche, Cat\# 1836145) per $50 \mathrm{ml}$ of conversion buffer. The resulting $10 \%$ normal $\mathrm{BH}$ in conversion buffer was used as the substrate in PMCA reactions. To prepare seeds, $10 \%$ scrapie $\mathrm{BH}$ in PBS was diluted 100-fold in the conversion buffer and $10 \mu \mathrm{l}$ of the dilution were used to seed $90 \mu \mathrm{l}$ of NBH in PMCA. Samples in $0.2 \mathrm{ml}$ thin-wall PCR tubes were placed in a floating rack inside Misonix-3000 cup-horn sonicator, filled with $300 \mathrm{ml}$ water. Two coils of rubber tubing attached to a circulating water bath were installed for maintaining $37^{\circ} \mathrm{C}$ inside the sonicator chamber. The standard sonication program consisted of $40 \mathrm{~s}$ sonication pulses delivered at $80 \%$ efficiency applied every hour during a 48-h period.

Brain homogenates from the first passage animals were subjected to a single round of PMCA in an attempt to increase the sensitivity of detection. In this case, the $\mathrm{BH}$ served both as seed and as substrate for the amplification. A half brain from each animal (non-perfused) was homogenized in the conversion buffer and aliquots of $100 \mu \mathrm{l}$ were subjected to $40 \mathrm{~s}$ sonication pulses applied every hour during 48-h period as described above.

To analyze production of PK-resistant PrP material in PMCA, $15 \mu \mathrm{l}$ of the sample was supplemented with $2.5 \mu \mathrm{l}$ SDS and $2.5 \mu \mathrm{PK}$, to the a final concentration of SDS and PK of $0.25 \%$ and $50 \mu \mathrm{g} / \mathrm{ml}$, respectively, followed by incubation at $37^{\circ} \mathrm{C}$ for $1 \mathrm{~h}$. The digestion was terminated by addition of SDS-sample buffer and boiling for $10 \mathrm{~min}$. Samples were loaded onto NuPAGE 12\% BisTris gels, transferred to PVDF membrane, and detected with $3 \mathrm{~F} 4$ antibody.

\section{Histopathological studies}

Tissues from three diseased hamsters from the second passage of the NBH-annealed fibril preparation, four same age controls that had been inoculated with BHs from the uninoculated same age controls of the first passage, and three uninoculated age-matched controls from the cohort of animals used for the second passage were examined. Formalin fixed brain halves divided at the midline (right hemisphere), spinal cord, and internal organs, including tongue, testis, fat tissue, intestine at different levels, stomach, spleen, liver, pancreas, kidney, bladder, adrenals, heart, lung, trachea, skin, hind leg muscle, salivary and mesenteric lymph nodes, thymus, and sciatic nerve were processed for conventional stains including hematoxylin-eosin and luxol fast blue-nuclear fast red, Alcian-blue and Periodic acid Schiff (PAS), as well as for immunohistochemistry for PrP, using the monoclonal anti-PrP antibody 3F4 (1:1000, Covance, Berkeley, CA, USA), and monoclonal anti-glial fibrillar acidic protein (GFAP; 1:3000, Dako, Glostrup, Denmark). Blocks were treated in formic acid (96\%) prior to embedding in paraffin. For detection of disease-associated $\operatorname{PrP}$, we applied a pretreatment of 30-min hydrated autoclaving at $121{ }^{\circ} \mathrm{C}$ followed by $5 \mathrm{~min}$ in $96 \%$ formic acid. We evaluated all tissues for the presence of inflammation and PrP immunoreactivity, and the brain for the presence of spongiform change and degree of gliosis. Degree of spongiform change, neuronal loss and gliosis, and intensity of PrP immunostaining was semiquantitatively evaluated ( 0 none, 1 mild, 2 moderate, and 3 severe). Lesion profiles were obtained by averaging scores of spongiform change, neuronal loss, and gliosis for each anatomical region and animal group [25]. For ultrastructural investigation, small samples of spinal cord periaqueductal regions obtained by needle biopsy from the paraffin block, were deparaffinized, fixed in glutaraldehyde and embedded in epoxy resin. Semi-thin sections were cut at a thickness of $0.3-0.5 \mu \mathrm{m}$ and were stained with toluidine blue. Ultrathin sections of 80-90 nm were analyzed with a Zeiss electron microscope. In addition, we compared brain pathology with three Syrian hamsters (Harlan, WI) that had been infected by intracranial injection with $10 \%(\mathrm{w} / \mathrm{v})$ scrapie brain homogenate (263K strain) and that showed clinical evidence of disease.

\section{Results}

De novo generation of $\operatorname{PrP}^{\mathrm{Sc}}$ in wild-type animals

Full-length golden Syrian hamster rPrP was converted into amyloid fibrils and subjected to annealing prior to inoculation $[5,6]$. Previously we showed that annealing (heating to $80^{\circ} \mathrm{C}$ in the presence of normal brain homogenate $(\mathrm{NBH})$ ) extends the short proteinase $\mathrm{K}$ (PK)-resistant region in $\mathrm{rPrP}$ fibrils encompassing residues 152/162-231 to residues $\sim 97-231[5,6]$. The annealing was performed in the presence of NBH prepared from 25-day-old golden Syrian hamsters or with bovine serum albumin (BSA). The $\mathrm{NBH}$-assisted annealing produced a $16 \mathrm{kDa}$ PK-resistant band, whereas in BSA-assisted annealing a $16 \mathrm{kDa}$ band was not detectable (Fig. 1a). All experiments on expression and purification of $\mathrm{rPrP}$, its conversion into fibrils, annealing and preparation of inocula were performed in a facility and with equipment that have never been exposed to TSEs (concerns regarding possible cross-contamination are addressed in SI).

The NBH- and BSA-annealed rPrP fibrils were inoculated intracerebrally into golden Syrian hamsters along with the following controls: $\alpha$-rPrP-monomer mockannealed with NBH, mock-annealed NBH alone, and nonannealed rPrP fibrils. The flow chart outlining primary 
a

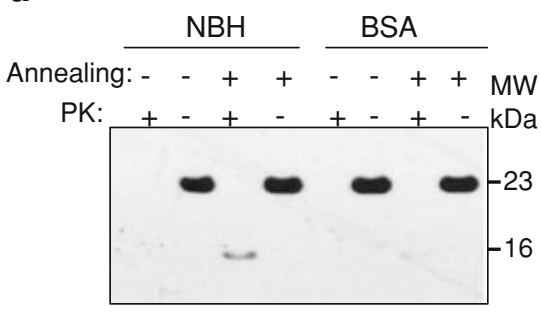

C \# $_{\frac{\pi}{\pi}}^{\frac{.}{\frac{1}{4}}}$

$2^{\text {nd }}$ passage $(\mathrm{BH}$ from \#36798)
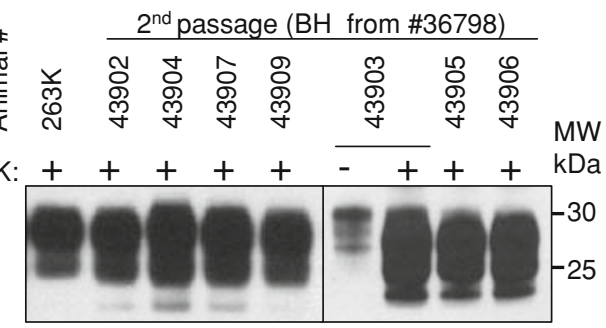

$\mathrm{PK}:+++++\overline{-t}++\mathrm{kDa}$

b \#

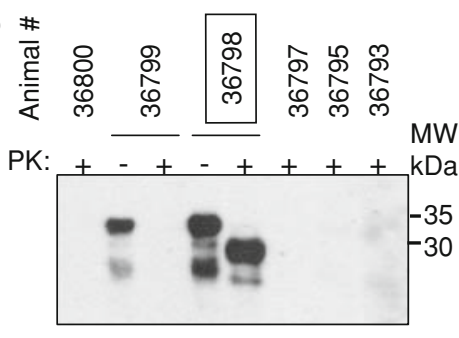

d $2^{\text {nd }}$ passage ( $\mathrm{BH}$ from \#36793)

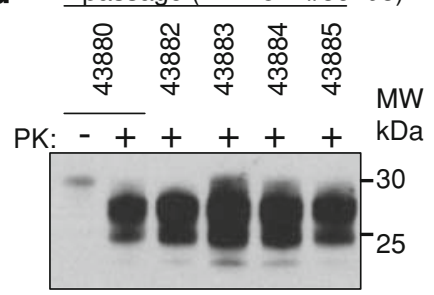

e

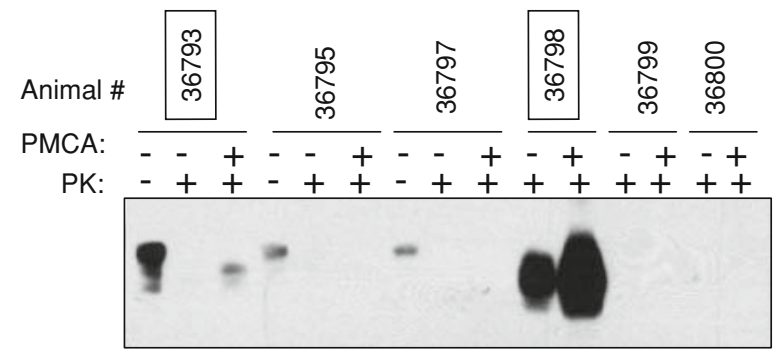

Fig. 1 De novo generation of $\operatorname{PrP}^{\mathrm{Sc}}$ in Syrian hamsters. a Western blotting of $\mathrm{rPrP}$ fibrils annealed in the presence of NBH or BSA. $\mathrm{NBH}$-annealed fibrils showed a PK-resistant product of $16 \mathrm{kDa}$. Undigested samples were loaded at $1 / 20$ th the amount of the digested samples. b Western blotting of BHs from animals inoculated with NBH-annealed fibrils. Animal \#36798 showed PK-resistant $\mathrm{PrP}^{\mathrm{Sc}}$ with a characteristic band shift. c Western blotting of BHs from animals inoculated with $\mathrm{BH}$ from animal \#36798. Without PK treatment, 1/10th of a sample was loaded. d Western blotting of BHs

inoculations and serial passages is presented in Fig. 2. None of the animals developed any signs of disease for up to 661 days after inoculation when they were euthanized (Table 1). One hamster inoculated with NBH-annealed fibrils (animal \#36798) showed PK-resistant PrP with a band-shift typical for $\operatorname{PrP}^{\mathrm{Sc}}$ (Fig. 1b). One animal inoculated with BSA-annealed rPrP amyloid (animal \#36787) also showed PK-resistance, however, with atypical, low molecular weight (LMW) PK-resistant bands (Fig. S1). No PK-resistant PrPs were found in animals inoculated with any of the controls or in uninoculated age-matched controls (Table 1, Fig. S2).

Brain homogenates (BHs) from six animals that represent three groups were selected for a second passage: (1) $\mathrm{BH}$ with $\mathrm{PrP}^{\mathrm{Sc}}$ (animal \#36798) and $\mathrm{BH}$ that lacked detectible $\operatorname{PrP}^{\mathrm{Sc}}$ (animal \#36793), both from the group inoculated with NBH-annealed fibrils; (2) BH with LMW PK-resistant (animal \#36787) and BH that lacks detectible PK-resistant PrPs (animal \#36788), both from the group from animals inoculated with 10\% BH from animal \#36793. Undigested sample was loaded at 1/10th the amount of the digested samples. e Western blotting of BHs from animals inoculated with $\mathrm{NBH}$-annealed fibrils. BHs were subjected to a single round of PMCA that consisted of 40-s sonication pulses applied every hour during a 48-h period, where each sample was its own substrate. Samples in a, b, e were treated with $50 \mu \mathrm{g} / \mathrm{ml} \mathrm{PK}$, and samples in $\mathbf{c}$ and $\mathbf{d}$ with $20 \mu \mathrm{g} / \mathrm{ml}$ PK. 3F4 antibody was used for $\mathbf{a}, \mathbf{c}-\mathbf{e}$ and R1 antibody was used for $\mathbf{b}$
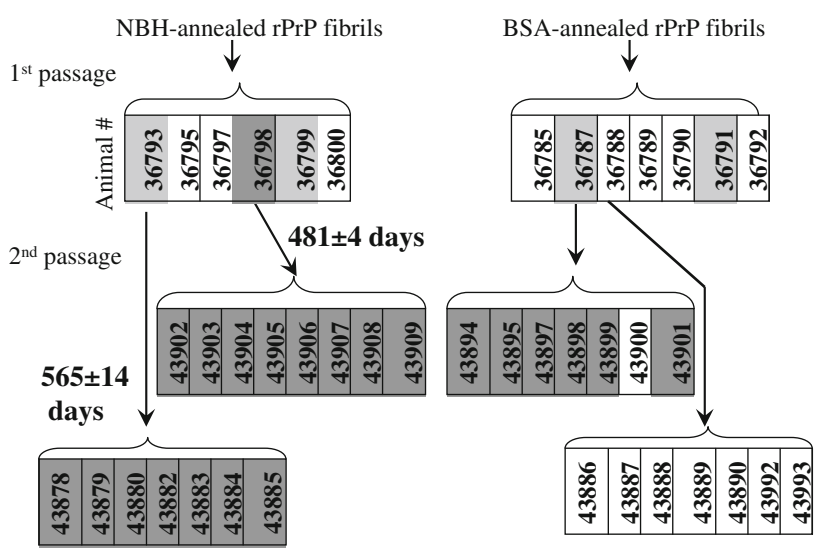

Fig. 2 A flow chart outlining the serial passages of NBH- or BSAannealed $\mathrm{rPrP}$ fibrils. Animal numbers are shown in the boxes. The animals that produced $\mathrm{PrP}^{\mathrm{Sc}}$ detectible by Western blotting are in dark gray boxes, and the animals where $\operatorname{PrP}^{\mathrm{Sc}}$ was detected after a single round of PMCA are in light gray boxes. Animal \#36787 showed atypical, low molecular weight PK-resistant bands 
Table 1 Bioassay of rPrP amyloid fibrils in golden Syrian hamsters

\begin{tabular}{|c|c|c|c|c|}
\hline Inoculum & $n_{\mathrm{s}} / n_{\mathrm{t}}^{\mathrm{a}}$ & $n_{\text {PK-res }} / n_{\mathrm{t}}^{\mathrm{b}}$ & $\begin{array}{l}\text { Clinical onset } \\
\text { (days PI) }\end{array}$ & $\begin{array}{l}\text { Euthanized } \\
\text { at days PI }\end{array}$ \\
\hline NBH-annealed rPrP fibrils & $0 / 6$ & $3 \%$ & - & 661 \\
\hline BSA-annealed rPrP fibrils & $0 / 7$ & $2 \%$ & - & 661 \\
\hline None (age-matched controls) & $0 / 9$ & $0 / 9$ & - & $661^{\mathrm{f}}$ \\
\hline Mock-annealed NBH & $0 / 7$ & $0 / 7$ & - & 660 \\
\hline$\alpha$-rPrP-monomer mock-annealed with $\mathrm{NBH}$ & $0 / 8$ & $0 / 8$ & - & 660 \\
\hline rPrP-fibrils ${ }^{c}$ & $0 / 7$ & $0 / 7$ & - & $690,704,5$ at 732 \\
\hline 2nd Passage: $10 \% \mathrm{BH}$ from age-matched control \#1 & $0 / 5$ & $0 / 5$ & - & 661 \\
\hline 2nd Passage: $10 \% \mathrm{BH}$ from age-matched control \#2 & $0 / 5$ & $0 / 5$ & - & 661 \\
\hline 2nd Passage of NBH-annealed fibrils (from \#36798) & $8 / 8$ & $8 / 8$ & $481 \pm 4$ & $\begin{array}{l}523,561,561,568 \\
\quad 574,586,586,601\end{array}$ \\
\hline 2nd Passage of NBH-annealed fibrils (from \#36793) & $7 / 7$ & $7 / 7$ & $565 \pm 14$ & 1 at 655,6 at 661 \\
\hline 2nd Passage of BSA-annealed fibrils (LMW PK-resistant, from \#36787) & $0 / 7$ & $6 / 7$ & - & 661 \\
\hline 2nd Passage of BSA-annealed fibrils (PK-sensitive, from \# 36788) & $0 / 7$ & $0 / 7$ & - & 661 \\
\hline
\end{tabular}

${ }^{a}$ Number of animals with clinical signs over the total number of animals survived to the end of the experiment

b Number of animals with PK-resistant PrP in BHs over the total number of animals survived to the end of the experiment

c The rPrP fibrils (S-type) were produced as previously described [22]

${ }^{\mathrm{d}}$ In one $\mathrm{BH} \mathrm{PK}-$ resistant $\mathrm{PrP}^{\mathrm{Sc}}$ was found by PK digestion assay, and in two additional BHs PK-resistant $\mathrm{PrP}^{\mathrm{Sc}}$ appeared after a single round of PMCA

e One BH showed PK-resistant PrP with atypical low molecular weight bands; this and one additional BH showed PK-resistant PrP ${ }^{\mathrm{Sc}}$ after a single round of PMCA

f Age-matched control animals were not inoculated, but euthanized at the age matching the age of corresponding inoculated groups

inoculated with BSA-annealed fibrils; and (3) two uninoculated age-matched controls (Table 1, Fig. 2). All hamsters inoculated with $\mathrm{BH}$ of animal \#36798 from the $\mathrm{NBH}-a n n e a l e d$ group showed clinical signs of disease at $481 \pm 4$ days after inoculation (Table 1). The clinical signs and progression were highly unique (detail description is in SI, movie clips 1-4). The clinical disease progressed unusually slowly, therefore one animal (\#43902) was euthanized 42 days after clinical onset to establish whether this was indeed a TSE infection. The conditions of the remaining animals deteriorated very slowly and all were euthanized from 80 to 120 days after onset of clinical symptoms before any had reached a terminal moribund stage. All BHs from this group including animal \#43902 showed large amounts of PK-resistant $\mathrm{PrP}^{\mathrm{Sc}}$ (Fig. 1c). As judged from Western blotting, the amount of PK-resistant $\mathrm{PrP}^{\mathrm{Sc}}$ exceeded by several fold the amount of $\mathrm{PrP}^{\mathrm{Sc}}$ in $263 \mathrm{~K}$-inoculated hamsters at the terminal stage (Fig. S3).

Surprisingly, all animals inoculated with $\mathrm{BH}$ of animal \#36793 that received NBH-annealed fibrils also developed clinical disease, however, at an older age (565 \pm 14 days after inoculation, Table 1). This group had the same symptoms as those inoculated with the $\mathrm{BH}$ of animal \#36798 and showed similarly large amounts of PK-resistant $\mathrm{PrP}^{\mathrm{Sc}}$ in their brains (Fig. 1d). Puzzled by these results, we went back and analyzed PK-resistance in BHs of animals that received NBH-annealed fibrils again. Prior to PKdigestion, however, $10 \%$ BHs were subjected to a sonication procedure similar to a single round of protein misfolding cyclic amplification (PMCA) that consisted of 48 cycles. The $\mathrm{BH}$ of animal \#36793 that was selected for the second passage showed a small amount of PK-resistant $\mathrm{PrP}^{\mathrm{Sc}}$ after sonication (Fig. 1e, Fig. S4a). This small, initially undetectable quantity of PK-resistant $\mathrm{PrP}^{\mathrm{Sc}}$ cannot be due to horizontal transmission of prions from the animal \#36798, because these two animals were housed in different cages. When the conditions for PMCA reaction were optimized for the newly generated TSE strain, two additional BHs (\#36799 and \#36791), one from the group inoculated with NBH-annealed fibrils and another from the group inoculated with BSA-annealed fibrils showed PK-resistant $\mathrm{PrP}^{\mathrm{Sc}}$ (Fig. S4b). In summary, 3 out of 6 animals inoculated with $\mathrm{NBH}-a n n e a l e d$ fibrils showed infectivity/ $\mathrm{PrP}^{\mathrm{Sc}}$ as detected by either second passage or by a single-round PMCA. PMCA has been used to generate prion infectivity de novo from normal brain homogenate but only after 10 rounds of 240 sonication cycles per round. After 50 days of sonication only $1 \mathrm{BH}$ out of 10 showed $\mathrm{PrP}^{\mathrm{Sc}}$ [2]. In contrast, we used only a single round of 48 cycles, where seed and substrate were in the same homogenate, and where our objective was to increase sensitivity of detection. There was no possibility of de novo synthesis as born out by controls. None of the BHs from any of the negative control groups 
that included uninoculated age-matched controls, second passages of two uninoculated age-matched controls, animals inoculated with mock-annealed $\mathrm{NBH}$, or with $\alpha$-rPrP-monomer mock-annealed with NBH produced any PK-resistant PrP after PMCA (Table 1, Figs. S2, S4b).

None of the hamsters from the second passage of BSAannealed fibrils developed clinical disease before they were euthanized at 661 days after inoculation. Despite the lack of clinical signs, 6 out of 7 hamsters inoculated with LMW PK-resistant BH showed PK-resistant PrP in amounts similar to those found at the terminal stage of $263 \mathrm{~K}$-inoculated hamsters and with a band-shift typical for $\operatorname{PrP}^{\mathrm{Sc}}$ (Fig. S5a).
Unique biochemical features of the de novo generated prions

Biochemical assays were employed to characterize the newly generated TSE strain that was designated SSLOW (Synthetic Strain Leading to OverWeight). While the $\operatorname{PrP}^{\mathrm{Sc}}$ glycosylation pattern of SSLOW appeared to be similar to that of $263 \mathrm{~K}$, the $\operatorname{PrP}^{\mathrm{Sc}} \mathrm{PK}$-resistant core was slightly shorter in SSLOW (by $\sim 0.5-1 \mathrm{kDa}$ ) than in $263 \mathrm{~K}$, as judged from the mobility after deglycosylation by PGNase (Fig. 3a). The slightly shorter size was a characteristic feature of $\mathrm{PrP}^{\mathrm{Sc}}$ produced in the first and second passage of NBH-annealed fibrils (Fig. 3a). The conformational

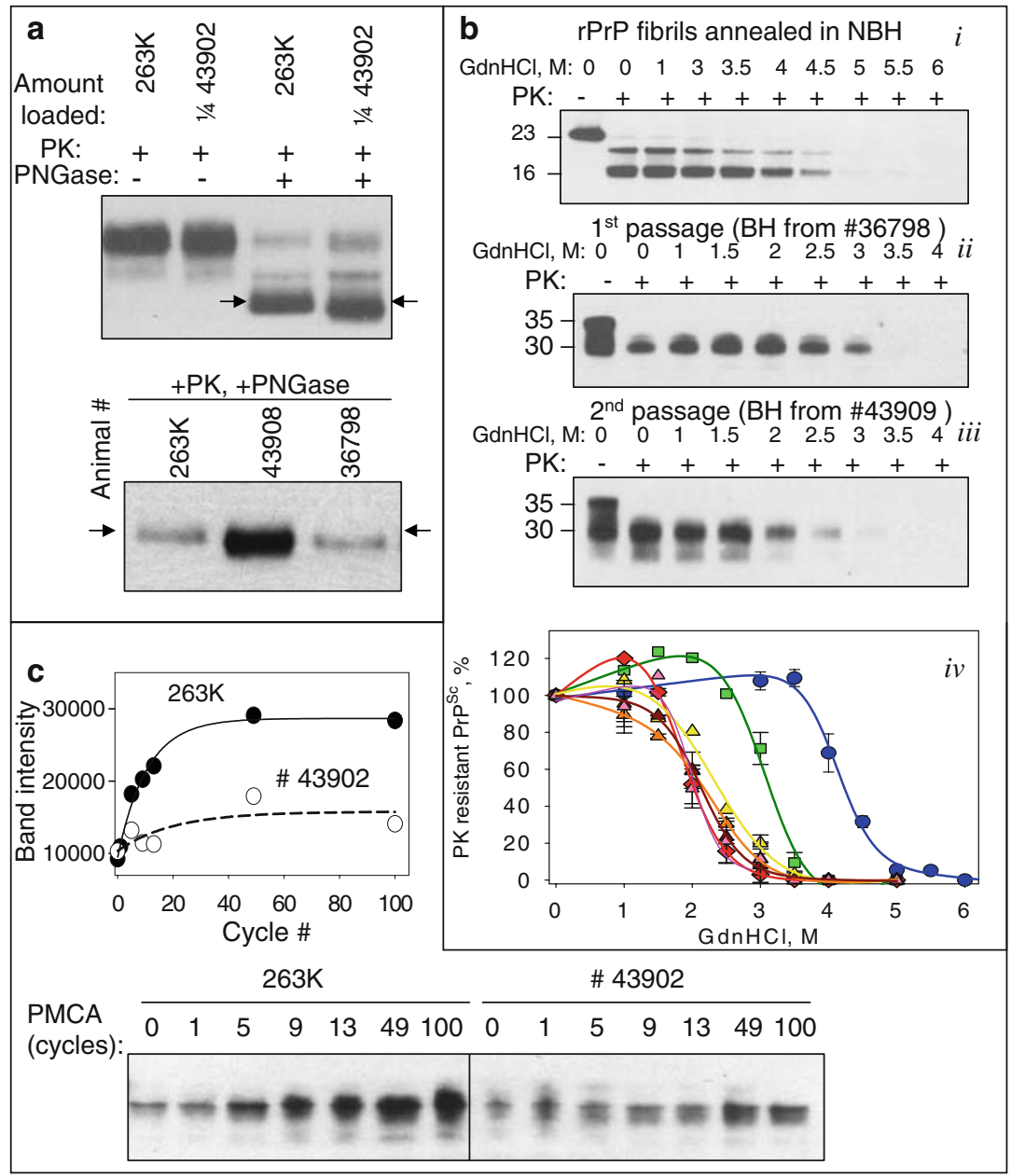

Fig. 3 Biochemical characterization of the de novo generated prions. a BHs from the first (animal \#36798) or second passage (animals \#43902 and \#43908) of NBH-annealed fibrils were treated with PK and PNGase and analyzed by Western blotting. Arrows indicate the center of the deglycosylated $\mathrm{PrP}^{\mathrm{Sc}}$ from $263 \mathrm{~K}$-inoculated hamsters. $\mathrm{BH} \# 43902$ was loaded at $1 / 4$ th of the amount of $263 \mathrm{~K} \mathrm{BH}$. b Western blotting and the conformational stability profiles $(i v)$ of the $\mathrm{GdnHCl}-$ induced transitions of the $\mathrm{NBH}$-annealed fibrils $(i)$, or $\mathrm{PrP}^{\mathrm{Sc}}$ from first (ii) or second (iii) passage of NBH-annealed fibrils. NBH-annealed fibrils (blue circles), $\operatorname{PrP}^{\mathrm{Sc}}$ from first passage (green square, animal
\#36798), second passage (yellow, orange, brown, or pink trianglesanimals \#43902, 43904, 43908, or 43909), or 263K-inoculated animals (red diamonds). Each $\mathrm{BH}$ was loaded onto the gel twice and the data were averaged. $\mathrm{c}$ The amplification kinetics for $263 \mathrm{~K}$ or $\mathrm{PrP}^{\mathrm{Sc}}$ from second passage of $\mathrm{NBH}$-annealed fibrils ( $\left.\mathrm{BH} \# 43902\right)$ as monitored by Western blotting during a single round of PMCA that consisted of 100 cycles. Samples in $\mathbf{a}, \mathbf{b}$ ( $i i$ and $i i i)$ were treated with $20 \mu \mathrm{g} / \mathrm{ml} \mathrm{PK}$; samples in c with $50 \mu \mathrm{g} / \mathrm{ml} \mathrm{PK}$; and samples in b $(i)$ with $2 \mu \mathrm{g} / \mathrm{ml}$ PK 
stability assay revealed that the resistance of SSLOW to GdnHCl-induced denaturation decreased gradually over the course of serial transmission. The $[\mathrm{GdnHCl}]_{1 / 2}$ values were $4.1 \pm 0.05 \mathrm{M}, 3.0 \pm 0.1 \mathrm{M}$, and $2.0 \pm 0.2 \mathrm{M}$ for the $\mathrm{NBH}$-annealed fibrils used for inoculations, $\mathrm{PrP}^{\mathrm{Sc}}$ from the first passage (animal \# 36798), and $\mathrm{PrP}^{\mathrm{Sc}}$ from the second passage, respectively (Fig. 3b). After the second passage, the $[\mathrm{GdnHCl}]_{1 / 2}$ values for SSLOW were similar to that of 263K (Fig. 3b). Despite similar conformational stabilities, SSLOW had a much longer incubation time than $263 \mathrm{~K}$. Furthermore, the yield of in vitro replication of SSLOW was persistently lower than that of $263 \mathrm{~K}$, as judged from the kinetics of $\operatorname{PrP}^{\mathrm{Sc}}$ amplification measured within a single round of PMCA reactions (Fig. 3c). The resistance of $\mathrm{PrP}^{\mathrm{Sc}}$ to proteolytic digestion was similar for SSLOW and $263 \mathrm{~K}$ as evaluated by the assays conducted at increasing concentrations of PK (Fig. S6).

The deglycosylation with PGNase of the PK-resistant PrP from the second passage of BSA-annealed fibrils revealed that the PK-resistant core was similar to that of SSLOW strain being slightly shorter than the deglycosylated $\mathrm{PrP}^{\mathrm{Sc}}$ from $263 \mathrm{~K}$ (Fig. S5b). While the similarity in PK-resistant core suggests that the same strain is emerging in animals inoculated with BSA-annealed fibrils, it remains to be determined whether a clinical disease will appear in a third passage of BSA-annealed fibrils.

\section{SSLOW strain displays unique neuropathological} profile

Histopathological studies of hamsters from the second passage of NBH-annealed fibrils revealed characteristic signs of TSE infection including spongiform degeneration, neuronal loss, reactive astrogliosis, and deposition of disease-associated PrP in the brains and spinal cords (Fig. 4, Figs. S7, S8). No inflammatory changes or immunoreactivity for disease-associated $\operatorname{PrP}$ were found in any peripheral tissues examined (complete list of tissues is in Methods). Spongiform change, neuronal loss, and reactive astrogliosis were uniformly prominent in the thalamus, caudate-putamen, brain stem, spinal cord, and deeper layers of frontal cortex. However, they were very mild in the hippocampus and cerebellum (Fig. 4a, c-e, Fig. S7). In contrast to animals infected with SSLOW strain, the brains of $263 \mathrm{~K}$-infected hamsters showed uniform involvement of the cerebellum, hippocampus and all layers of the frontal cortex (Fig. 4a, c-e, Fig. S7).

The distribution and pattern of PrP immunostaining were remarkably different for SSLOW- and 263K-infected brains (Fig. 4b-e, Fig. S7). While both strains showed synaptic immunoreactivity for PrP deposits, SSLOW-infected animals were characterized by more intensive perineuronal deposits and large plaques with a diameter of $20-60 \mu \mathrm{m}$ in the subpial, periventricular, and periaqueductal subependymal regions (Fig. 4f-h, Fig. S7, S8). In contrast, $263 \mathrm{~K}$-infected brains showed focal patchy or smaller plaque-like deposits with a diameter of 5-15 $\mu \mathrm{m}$ in gray and white matter structures. Both strains displayed immunoreactivity in subependymal regions, whereas perivascular $\operatorname{PrP}$ deposits were found only in hamsters infected by the $263 \mathrm{~K}$ strain. While synaptic and perineuronal immunoreactivity showed anatomical variability between SSLOW-infected animals, the most striking histopathological feature of SSLOW strain was the uniform deposition of large plaques in the subpial, periventricular, and periaqueductal subependymal regions spanning all locations including brain and spinal cord (Fig. 4, Figs. S7, S8). These plaques stained with Alcian blue and with PAS at their borders (Fig. S8). Electron microscopy revealed that these plaques consist of a loose meshwork of randomly orientated filaments of 10 $25 \mathrm{~nm}$ in diameter (Fig. 4j, k). Animals from control groups lacked any spongiform degeneration or deposition of disease-associated PrP (Fig. S9).

\section{Discussion}

The current studies demonstrate that recombinant PrP in its fibrillar $\beta$-sheet rich conformation subjected to annealing induces a transmissible form of prion disease in wild-type animals. In the first passage, NBH-annealed fibrils were found to seed formation of $\operatorname{PrP}^{\mathrm{Sc}}$ in hamster brains, but failed to cause clinical disease, which was only observed at the second passage. The lack of symptomatic disease by end of life is consistent with a very slow replication of $\mathrm{PrP}^{\mathrm{Sc}}$ and that the newly emerged strain is intrinsically slow. Previous work has shown that the incubation period of a prion disease can exceed the life span of an animal even when induced by natural prion strains [13-16, 19]. In classical studies by Dickinson et al. [13], mice inoculated with high titers of certain strains of mouse-adapted scrapie prions remained free of clinical symptoms for their entire life span despite histopathological changes and accumulation of $\operatorname{PrP}^{\mathrm{Sc}}$ in their brains at the end of their life span. Because incubation time, long or short, is an intrinsic function of TSE strain, it cannot be used to deduce the concentration of infectious material in the inoculum without more knowledge of the dose response. A number of lines of evidence in the current study suggest that the newly generated strain that we call SSLOW is intrinsically very slow. When the incubation time exceeds the life span of the animal it may be difficult to determine the actual transmission rate because one can detect only those infections that have progressed to a detectable endpoint by the end of life. To increase the sensitivity at the endpoint, we employed a single-round PMCA. Using this procedure, 
Fig. 4 SSLOW strain displays unique neuropathological profile. a, b Lesion profile (a) and PrP immunopositivity score (b) in hamsters inoculated with SSLOW (2nd passage of NBHannealed fibrils) or $263 \mathrm{~K}$. The lesion profile was obtained by averaging the scores for spongiform change, neuronal loss, and gliosis for three animals within each group. c-e Comparison of spongiform changes in the hippocampus (c), thalamus (d), or cerebellum (e) stained with hematoxylin and eosin (upper panels) or antiPrP 3F4 antibody (lower panels) in a representative hamsters inoculated with SSLOW (animal \# 43902, left panels) or 263K (right panels). f Lowmagnification $(\times 20)$ overview of frontal cortex (enlarged at $\times 100$ in right upper inset) and caudate-putamen (enlarged at $\times 100$ in right lower inset) using immunostaining for diseaseassociated PrP. g, h Highmagnification images $(\times 400)$ of PrP immunoreactive plaques in the periventricular subependymal region (g), and synaptic and perineuronal $\operatorname{PrP}$ immunoreactivity in the frontal cortex (h). i Overview of PrP immunostaining $(\times 50)$ of spinal cord demonstrates prominent plaque deposition (enlarged right lower inset) in the midline and in periaqueductal region. $\mathbf{j}, \mathbf{k}$ Electron microscopy of a plaque from the periaqueductal region from the spinal cord (scale bar $10 \mu \mathrm{m}$ in $\mathbf{j}$ or $0.4 \mu \mathrm{m}$ in $\mathbf{k}$ )

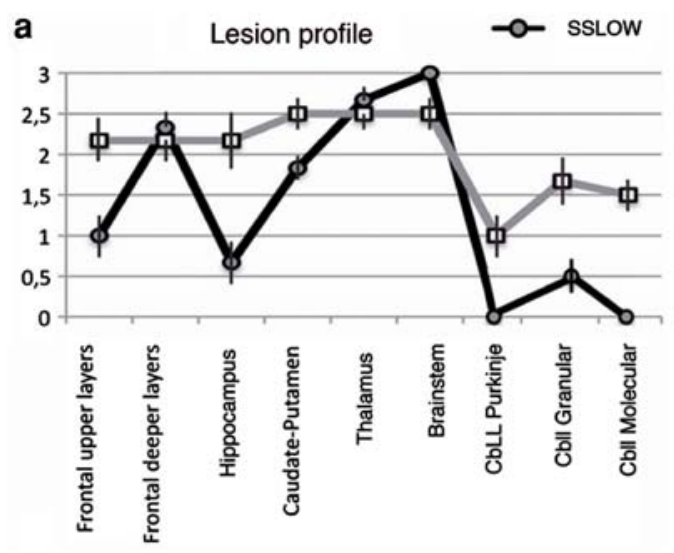

b - - sc $263 \mathrm{~K} \quad$ PrP immunoreactivity score


$\mathrm{PrP}^{\mathrm{Sc}}$ was detected in two additional animals inoculated with NBH-annealed fibrils. We do not know whether or not all of the animals from the first passage would have proved to be infected, had we carried all of them forward in a second passage.

The apparent $50 \%$ efficiency of infection in animals inoculated with NBH-annealed fibrils could be attributed to several factors. First, because rPrP fibrils lack a GPI-anchor, they do not attach to the cell surface as efficiently as authentic $\operatorname{PrP}^{\mathrm{Sc}}$ and, therefore, might display reduced ability to invade cells and seed prion replication. Second, rPrP fibrils and $\operatorname{PrP}^{\mathrm{Sc}}$ appear to have fundamentally different global structures [29]. Because $\operatorname{PrP}^{\mathrm{C}}$ might not be fully compatible with the structure of $\mathrm{rPrP}$ fibrils, seeding of $\mathrm{PrP}^{\mathrm{C}}$ conversion with rPrP fibrils might proceed through slow conformational adaptation [3]. The gradual change in conformational stability during serial transmission observed here supports the hypothesis of conformational adaptation. Previous studies 
on synthetic mouse prions revealed a similar trend, where conformational stability of the de novo generated synthetic strains was found to decrease gradually during their serial transmission $[9,21]$. The conformational adaptation could involve a conformational switch within individual fibrils [23], where $\operatorname{rPrP}$ fibrils with one global structure might recruit $\operatorname{PrP}^{\mathrm{C}}$, elongate and give a rise to $\operatorname{PrP}^{\mathrm{Sc}}$ with different global structure. Third, as postulated by the "unified theory" of prion propagation [28], efficient replication of prions appears to require cellular cofactors, such as RNAs [11, 12]. The $50 \%$ efficiency of the infection in the first passage indicates that the annealing protocol might not yet faithfully mimic the interaction of $\mathrm{rPrP}$ with a co-factor for generating authentic $\operatorname{PrP}^{\mathrm{Sc}}$ in vitro. The fact that $\mathrm{PrP}^{\mathrm{Sc}}$ was found in both animal groups, inoculated either with $\mathrm{NBH}-$ or BSAannealed fibrils, suggests that the role of NBH-annealing is non-specific and that, perhaps, it plays a role of a stabilizer. By analogy, numerous viruses must be stabilized with milk, serum, albumin, or gelatin to survive inoculation.

The 50\% transmission at first passage is also consistent with a very low titer of the active species in the original inoculum. However, if one assumes that only a small subpopulation of fibrils is infectious, it would be difficult to explain a correlation between conformational stability of $\mathrm{rPrP}$ fibrils, which is a bulk property of fibril preparations, and the incubation times to disease observed in the previous study on synthetic prions [9].

One of the most remarkable features of the prion disease caused by SSLOW strain is its long clinical duration after the first clinical signs are observed. In fact, the actual duration to a death endpoint is not known as the animals, while seriously compromised, were still mobile, eating and drinking at the time of euthanasia. It is conceivable that animals may have endured longer than 120 days after first clinical signs, if they had not been euthanized. Such a long duration is more typical of human and large animal TSEs, than those of rodents. The slow progression is surprising considering that large amounts of $\operatorname{PrP}^{\mathrm{Sc}}$ were already present at the initial stages of clinical disease. The slow progression may be attributable to the very peculiar pattern of deposition of $\operatorname{PrP}^{\mathrm{Sc}}$ which was found predominantly in the form of large plaques. The $\operatorname{PrP}^{\mathrm{Sc}}$ deposition patterns and brain histopathology differed prominently between SSLOW and $263 \mathrm{~K}$ or other hamster strains. One of the most intriguing features of the SSLOW strain was the very minor involvement of the cerebellum highlighting a unique neurotropism of this strain. Very mild $\mathrm{PrP}^{\mathrm{Sc}}$ deposition in the cerebellum correlated well with apparent lack of the severe motor dysfunctions typical for 263K. The sparing of the hippocampus in SSLOW is intriguing and reminiscent of sparing of the hippocampus in the most common subtype (MM/MV-1) of human sporadic Creutzfeldt-Jakob disease (CJD) [7, 25]. In contrast, the large subpial and subependymal plaques were distinct not only from plaque-like deposits in $263 \mathrm{~K}$ scrapie of hamsters but also from human TSEs with plaques including the Kuru plaque variant (MV-2) of sporadic CJD or GerstmannStraussler-Scheinker disease.

In second passage of NBH-annealed fibrils, the two groups of animals inoculated with BHs from \#36793 and \#36798 showed different incubation times to onset of disease. This difference can be attributed to a lower infectivity titer in $\mathrm{BH}$ from \#36793, consistent with the substantially lower amount of PK-resistant $\mathrm{PrP}^{\mathrm{Sc}}$ in this brain. As judged from clinical signs and biochemical assays, however, the same strain appears to have emerged in both groups. Remarkably, in a second passage of BSA-annealed fibrils, PK-resistant PrP showed a band shift identical to that of SSLOW $\operatorname{PrP}^{\mathrm{Sc}}$ raising the possibility that the same stain might emerge in serial passages of two independently prepared inoculums: NBH- or BSA-annealed fibrils. The third passage of NBH- and BSAannealed fibrils should clarify this question.

The conformational stability of SSLOW $\mathrm{PrP}^{\mathrm{Sc}}$ that formed in a second passage was very similar to that of $263 \mathrm{~K}$, yet these two strains showed markedly different incubation times arguing that factors other than conformational stability also contribute to the rate of prion replication. Nevertheless, the substantially longer incubation time for SSLOW correlated well with the lower yield of SSLOW replication in PMCA as compared to 263K (Fig. 3c).

Much of the public health risk from TSE diseases derives from their long asymptomatic incubation times. During this period infected humans (or animals) appear normal and their underlying disease is currently undetectable. Such individuals pose a real risk of spreading the infection through invasive medical procedures or tissue or blood donation. Variant CJD infections of humans heterozygous at the codon 129 of the prion gene may present such a risk [18]. The SSLOW strain offers an experimental model to investigate this problem.

In pursuing the objective of generating synthetic prions in vitro, it is useful to consider how prions originate in the natural environment. Spontaneous occurrence of transmissible prion disease has never been documented in wild-type rodents including golden Syrian hamsters or wild-type mice, nor has prion disease occurred upon serial inoculations of brain homogenates from old to young animals [17]. All experimental hamster or mouse strains have been derived by serial transmission of TSE agents from species with substantially longer life span than rodents. While other neurodegenerative diseases can be triggered as a result of proteostasis deficiency at old age [1], the genesis of transmissible prions appears to require a very specific trigger and/or conditions that are much more peculiar than imbalance of proteostasis. Surprisingly, the current study suggests that transmissible prion disease can be induced by a preparation of $\mathrm{rPrP}$ fibrils, which appear to be 
fundamentally different with respect to their global structure from $\mathrm{PrP}^{\mathrm{Sc}}$. One can speculate that only partial or distant similarities between fibrillar $\mathrm{rPrP}$ and $\operatorname{PrP}^{\mathrm{Sc}}$ structures may be sufficient for triggering conversion of $\mathrm{PrP}^{\mathrm{C}}$ into $\mathrm{PrP}^{\mathrm{Sc}}$ by fibrillar $\mathrm{rPrP}$. We do not know whether the emergence of highly efficient prion strains in nature occurs within a single life span or require evolution, adaptation, and selection of $\operatorname{PrP}^{\mathrm{Sc}}$ structures. Prion genesis in nature might require a series of transmissions from animal to animal as may have happened with Bovine Spongiform Encephalopathy. In this regard, it would be interesting to learn whether clinical disease appears in a third passage of BSA-annealed fibrils.

In summary, this work represents an important advance in prion science, in that (i) it is the first time that a wild-type recombinant PrP has been shown to be able to induce a TSE disease in a wild-type animal; (ii) the disease generated by the in vitro refolded $\mathrm{rPrP}$ while clearly recognizable as a TSE disease is unique in its clinical presentation, pathology, and biochemistry and may prove of great interest. Because of its unique features, the SSLOW strain may prove to be a valuable model for addressing a number of puzzling questions of prion biology: what factors determine prion neurotropism; how strain-specific $\mathrm{PrP}^{\mathrm{Sc}}$ structures control the rate of prion replication; why clinical disease could progresses so slowly despite large amounts of $\mathrm{PrP}^{\mathrm{Sc}}$ deposition; and the physical nature of toxic versus infectious PrP isoforms. Further studies on synthetic prions will likely provide unique insights into the genesis and evolution of natural prion strains.

Acknowledgments We thank Pamela Wright for editing the manuscript. This work was supported by University of Maryland Biotechnology Institute and Baltimore Research and Education Foundation. Parts of this work were done in the framework of the EU Network of Excellence NeuroPrion, Subproject PrioGen.

Open Access This article is distributed under the terms of the Creative Commons Attribution Noncommercial License which permits any noncommercial use, distribution, and reproduction in any medium, provided the original author(s) and source are credited.

\section{References}

1. Balch WE, Morimoto RI, Dillin A, Kelly JW (2008) Adapting proteostasis for disease intervention. Science 319:916-919

2. Barria MA, Mukherjee A, Gonzalez-Romero D, Morales R, Soto C (2009) De novo generation of infectious prions in vitro produces a new disease phenotype. PLOS Pathog 5:e1000421

3. Baskakov IV, Breydo L (2007) Converting the prion protein: what makes the protein infectious. Biochim Biophys Acta (Molecular Basis of Disease) 1772:692-703

4. Baskakov IV, Legname G, Baldwin MA, Prusiner SB, Cohen FE (2002) Pathway complexity of prion protein assembly into amyloid. J Biol Chem 277:21140-21148

5. Bocharova OV, Breydo L, Parfenov AS, Salnikov VV, Baskakov IV (2005) In vitro conversion of full length mammalian prion protein produces amyloid form with physical property of PrPSc. J Mol Biol 346:645-659

6. Bocharova OV, Makarava N, Breydo L et al (2006) Annealing PrP amyloid firbils at high temperature results in extension of a proteinase K resistant core. J Biol Chem 281:2373-2379

7. Budka H, Head MW, Ironside JW et al (2003) Prion disorders. In: Dickson DW (ed) Neurodegeneration: the molecular pathology of dementia and movement disorders. ISN Neuropath Press, Basel, pp 287-297

8. Castilla J, Saa P, Hetz C, Soto C (2005) In vitro generation of infectious scrapie prions. Cell 121:195-206

9. Colby DW, Giles K, Legname G et al (2009) Design and construction of diverse mammalian prion strains. Proc Acad Natl Sci USA 106:20417-20422

10. Deleault AM, Deleault NR, Harris BT, Rees JR, Supattapone S (2008) The effects of prion protein proteolysis and disaggregation on the strain properties of hamster scrapie. J Gen Virol 89:2642-2650

11. Deleault NR, Harris BT, Rees JR, Supattapone S (2007) Formation of native prions from minimal components in vitro. Proc Acad Natl Sci USA 104:9741-9746

12. Deleault NR, Lucassen RW, Supattapone S (2003) RNA molecules stimulate prion protein conversion. Nature 425:717-720

13. Dickinson AG, Fraser H, Outram GW (1975) Scrapie incubation time can exceed natural lifespan. Nature 256:732-733

14. Frigg R, Klein MA, Hegyi I, Zinkernagel RM, Aguzzi A (1999) Scrapie pathogenesis in subclinically infected B-cell-deficient mice. J Virol 73:9584-9588

15. Hill A, Collinge J (2003) Subclinical prion infection in human and animals. Br Med Bull 66:161-170

16. Hill AF, Collinge J (2003) Subclinical prion infection. Trends Microbiol 11:578-584

17. Hill AF, Joiner S, Linehan J et al (2000) Species-barrier-independent prion replication in apparently resistant species. Proc Natl Acad Sci USA 97:10248-10253

18. Kaski D, Mead S, Hyare H et al (2009) Variant CJD in an individual heterozygous for PRNP codon 129. Lancet 374:2128

19. Klein MA, Frigg R, Raeber AJ et al (1998) PrP expression in B lymphocytes is not required for prion neuroinvasion. Nat Med 4:1429-1433

20. Legname G, Baskakov IV, Nguyen H-OB et al (2004) Synthetic mammalian prions. Science 305:673-676

21. Legname G, Nguyen H-OB, Baskakov IV et al (2005) Strainspecified characteristics of mouse synthetic prions. Proc Natl Acad Sci USA 102:2168-2173

22. Makarava N, Baskakov IV (2008) The same primary structure of the prion protein yields two distinct self-propagating states. J Biol Chem 283:15988-15996

23. Makarava N, Ostapchenko VG, Savtchenko R, Baskakov IV (2009) Conformational switching within individual amyloid fibrils. J Biol Chem 284:14386-14395

24. Nishina K, Deleault NR, Mahal S et al (2006) The stoichiometry of host PrPC glycoforms modulates the efficiency of PrPSc formation in vitro. Biochemistry 45:14129-14139

25. Parchi P, Giese A, Capellari S et al (1999) Classification of sporadic Creutzfeldt-Jakob disease based on molecular and phenotypic analysis of 300 subjects. Ann Neurol 46:224-233

26. Prusiner SB (1982) Novel proteinaceous infectious particles cause scrapie. Science 216:136-144

27. Safar J, Wille H, Itri V et al (1998) Eight prion strains have $\mathrm{PrP}^{\mathrm{Sc}}$ molecules with different conformations. Nat Med 4:1157-1165

28. Weissmann C (1991) A "unified theory" of prion propagation. Nature 352:679-683

29. Wille H, Bian W, McDonald M et al (2009) Natural and synthetic prion structure from X-ray fiber diffraction. Proc Acad Natl Sci USA 106:16990-16995 